\title{
Uma alternativa no diagnóstico e monitoramento de Diabetes Mellitus a detecção via
}

\section{biomarcadores: uma revisão sistemática}

\author{
An alternative in the diagnosis and monitoring of Diabetes Mellitus to detection via biomarkers: a \\ systematic review
}

Una alternativa em el diagnóstico y seguimiento de la Diabetes Mellitus a la detección mediante

biomarcadores: una revisión sistemática

Recebido: 26/07/2021 | Revisado: 31/07/2021 | Aceito: 01/08/2021 | Publicado: 07/08/2021

\author{
Maria Clara Gomes Silva \\ ORCID: https://orcid.org/0000-0002-8469-1034 \\ Centro Universitário UniFacid Wyden, Brasil \\ E-mail: mc.claragomes.mc@hotmail.com \\ Jardes Figuerêdo do Rêgo \\ ORCID: https://orcid.org/0000-0001-8066-5077 \\ Centro Universitário UniFacid Wyden, Brasil \\ E-mail: jardesfr@hotmail.com
}

\begin{abstract}
Resumo
A Diabetes Mellitus é um distúrbio metabólico, de alta prevalência mundial, das células beta-pancreáticas. O diagnóstico atualmente ocorre por uso de testes glicêmicos combinados com a dosagem de alguns biomarcadores. Portanto, o trabalho objetiva analisar técnicas clínicas para diagnóstico e monitoramento terapêutico da diabetes mellitus, destacando formas alternativas e compensatórias. Foi realizada uma revisão sistemática via levantamento nas plataformas SCIELO, PUBMED e LILACS. As palavras-chave utilizadas foram combinadas nas línguas inglesa e portuguesa. O recorte temporal estabelecido inclui os anos de 2010 a 2021. Por fim, os dados foram organizados via fluxograma Prisma statement, quadro-resumo e escala de evidência GRADE. Na pesquisa, os autores relataram que a hemoglobina glicada não deve ser utilizada no rastreio de pré-diabetes, além de possuir interferentes. Quanto à albumina glicada, esta vai atuar como substituto e auxilia na deteç̧ão de complicações advindas da diabetes, da mesma maneira que o 1,5 anidroglucitol, além disso, pode detectar as chances de mortalidade pela doença. E por fim, o anti-GAD e o peptídeo $C$ auxiliam na detecção da diabetes tipo 1, avaliação da insulinoterapia e pesquisa de desenvolvimento de doenças autoimunes. Apesar dos biomarcadores apresentarem grandes benefícios, ainda é preferível o uso de testes glicêmicos. Enfim, por meio do estudo realizado é possível averiguar que os biomarcadores são metodologias alternativas que ainda podem ser aperfeiçoadas, são eficazes na detecção da doença, mas ainda não são utilizados desacompanhados ou aplicados efetivamente na diabetes gestacional. Além disso, não só alertam para os riscos de desenvolver complicações como são parâmetros cirúrgicos.
\end{abstract}

Palavras-chave: 1,5-Anidroglucitol; Diabetes Mellitus; Hemoglobina A Glicada; Peptídeo C.

\begin{abstract}
Diabetes Mellitus is a highly prevalent metabolic disorder of the beta-pancreatic cells worldwide. The diagnosis currently occurs by using glycemic tests combined with the dosage of some biomarkers. Therefore, this paper aims to analyze clinical techniques for diagnosis and therapeutic monitoring of diabetes mellitus, highlighting alternative and compensatory forms. A systematic review was carried out via survey in SCIELO, PUBMED and LILACS platforms. The keywords used were combined in English and Portuguese. The time frame established includes the years 2010 to 2021. Finally, data were organized via Prisma statement flowchart, summary table, and GRADE evidence scale. In their research, the authors reported that the glycated hemoglobin should not be used in screening for pre-diabetes, besides having interferences. As for the glycated albumin, it will act as a substitute and helps in detecting complications arising from diabetes, in the same way as the 1.5 anhydroglucitol, in addition, it can detect the chances of mortality from the disease. And finally, anti-GAD and C-peptide help in the detection of type 1 diabetes, evaluation of insulin therapy, and research into the development of autoimmune diseases. Although biomarkers have great benefits, the use of glycemic tests is still preferable. Finally, through this study it is possible to verify that biomarkers are alternative methodologies that can still be improved, are effective in detecting the disease, but are not yet used unattended or applied effectively in gestational diabetes. Moreover, they not only alert to the risks of developing complications but are also surgical parameteres.
\end{abstract}

Keywords: 1,5-Anhydroglucitol; Diabetes Mellitus; Glycated Hemoglobin A; C Peptide. 


\begin{abstract}
Resumen
La Diabetes Mellitus es un trastorno metabólico de alta prevalencia en las células beta del páncreas en todo el mundo. En la actualidad, el diagnóstico se realiza mediante el uso de pruebas glucémicas combinadas con la dosificación de algunos biomarcadores. Además, el trabajo tiene como objetivo analizar las técnicas clínicas para el diagnóstico y el seguimiento terapéutico de la diabetes mellitus, destacando las formas alternativas y compensatorias. Se realizó una revisión sistemática mediante encuesta en las plataformas SCIELO, PUBMED y LILACS. Las palabras clave utilizadas se combinaron en inglés y portugués. El marco temporal establecido incluye los años 2010 a 2021 . Por último, los datos se organizaron mediante el diagrama de flujo de declaraciones Prisma, la tabla de resumen y la escala de evidencia GRADE. Em la investigación, los autores relatan que la hemoglobina glicada no debe utilizarse en el rastreo de la prediabetes, además de poseer interferencias. En cuanto a la albúmina glicosilada, actuará como sustituto y ayuda a detectar las complicaciones de la diabetes, del mismo modo que el 1,5 anhidroglucitol, además, puede detectar las posibilidades de mortalidad por la enfermedad. Y, por último, los anti-GAD y el péptido C ayudan a detectar la diabetes de tipo 1, a evaluar el tratamiento con insulina y a investigar el desarrollo de enfermedades autoinmunes. Aunque los biomarcadores presentan grandes ventajas, sigue siendo preferible el uso de pruebas de glucemia. Finalmente, a través del estudio realizado, es posible comprobar que los biomarcadores son metodologías alternativas que aún pueden ser mejoradas, son eficaces en la detección de la enfermedad, pero aún no son utilizados de forma desatendida o aplicada de forma efectiva en la diabetes gestacional. Además, no solo alertan de los riesgos de desarrollar complicaciones, sino que también son parâmetros quirúrgicos.
\end{abstract}

Palabras clave: 1,5-Anhidroglucitol; Diabetes Mellitus; Hemoglobina A Glucada; Péptido C.

\title{
1. Introdução
}

A Sociedade Americana de Diabetes (2014) define Diabetes Mellitus (DM) como uma disfunção metabólica pancreática, ou seja, o órgão não consegue produzir e secretar a insulina de maneira normal. Logo, o resultado dessa disfunção é uma hiperglicemia constante. Os tipos mais clássicos da diabetes são o tipo 1 e tipo 2, sendo o primeiro tipo decorrente de mecanismos autoimunes, e o segundo de defeitos na secreção da insulina, caracterizando a resistência insulínica.

A diabetes faz parte do grupo das Doenças Crônicas Não Transmissíveis (DCNT), e é considerada um problema de saúde mundial. A taxa de mortalidade mundial dessa doença corresponde a 10,7\%. Logo, ressalta-se a importância do diagnóstico precoce e tratamento contínuo e rigoroso. Atualmente, a principal terapia consiste no uso do hormônio hipoglicemiante, insulina. Este também pode ser utilizado no rastreio da diabetes (Sociedade Brasileira de Diabetes, 20192020).

Nas situações em que o indivíduo é exposto a altas taxas de glicemia por tempo prolongado pode ocorrer processo conhecido por glicação proteica. Isso significa que açúcares quando em excesso, no caso a glicose, se conjugam com proteínas humanas. Como resultado, na DM ocorre a formação de hemoglobina glicada (HbA1c) e albumina glicada (AG), estas podendo atuar como excelentes biomarcadores, auxiliando na prevenção ou monitoramento da doença (Tavares et al., 2017).

Os avanços científicos permitiram os estudos de marcadores biológicos de enfermidades, como no caso da diabetes. Quando em exposição à hiperglicemia frequente, o corpo humano responde com alterações nos valores desses marcadores. Para Selvin et al. (2016), além da HbA1c, as flutuações glicêmicas também podem ser avaliadas via 1,5 anidroglucitol.

O 1,5 anidroglucitol (1,5 AG) é um monossacarídeo adquirido predominantemente através da dieta e é uma alternativa para detecção da diabetes mellitus. O que o diferencia dos demais marcadores é a forma como sua concentração se altera. Enquanto o HbA1c apresenta-se em altas quantidades na DM, o 1,5 AG vai se manifestar em baixas concentrações, isto é, ele é reabsorvido nos túbulos e pode competir com a glicose, quando em altas concentrações. Então, em estados hiperglicêmicos, o marcador estará em doses mínimas (Selvin et al., 2016).

Nos casos de pacientes com diabetes tipo 1, os biomarcadores específicos utilizados são os autoanticorpos, pois estes são característicos das doenças autoimunes. Por exemplo, o anticorpo contra descarboxilase do ácido glutâmico (Anti-GAD), um biomarcador com tempo de detecção prolongado. Estudos associam o aumento dos autoanticorpos com a perda funcional progressiva das células beta-pancreáticas. No entanto, a funcionalidade do anti-GAD ainda não é totalmente conhecida (Serfaty et al. 2010). 
No quesito diagnóstico, uns dos principais exames padrão na avaliação de DM são a glicemia em jejum e o teste de tolerância oral à glicose. A glicemia em jejum exige do paciente um jejum de 8 a 12 horas e resultados acima de $125 \mathrm{mg} / \mathrm{dL}$ alertam para um quadro de DM. No entanto, esse exame apresenta desvantagens quando utilizados para monitorar a doença, pois pode ocorrer falso-positivo no controle da glicemia, além de um jejum bastante prolongado. Por outro lado, o jejum não é exigido quando se dosa a HbA1c. Portanto, os testes glicêmicos são relevantes no diagnóstico enquanto os marcadores são eficientes na avaliação e monitoramento (Sá, Navas \& Alves, 2014).

Dessa maneira, estabelece-se que a pergunta da pesquisa baseia-se nas formas padrões de diagnóstico e acompanhamento da diabetes melllitus com base no uso de testes de níveis glicêmicos. Estes apresentam certas limitações no uso. Logo, é possível rastrear e supervisionar a diabetes através de biomarcadores e estes podem atuar como metodologia alternativa.

Em seguida, norteou-se uma questão para a pesquisa. Existem diversos exames utilizados na prática clínica quando se trata da diabetes mellitus. No entanto, o rastreio e a dosagem de biomarcadores apresentam diferenciais como a rapidez, facilidade de deteç̧ão e comodidade ao paciente.

O presente trabalho objetiva analisar as técnicas clínicas para diagnóstico e monitoramento terapêutico da diabetes mellitus, destacando formas alternativas e compensatórias.

Quanto aos objetivos específicos, visam avaliar alguns dos exames clínicos, na atualidade, voltados para a detecção e controle da diabetes mellitus; descrever como os biomarcadores são aplicados na rotina da prática clínica; abordar possíveis limitações e benefícios dos exames utilizados no diagnóstico da diabetes mellitus e oferecer uma alternativa no diagnóstico de diabetes através da dosagem de marcadores biológicos.

Flor e Campos (2017), através de um estudo transversal concluíram que a diabetes é de fato um problema com grande relevância. Atualmente, 8,3\% da população mundial é diabética, valor que corresponde a 382 milhões de pessoas. Estima-se que em 2035, o número de diabéticos chegue a 592 milhões.

Em 2013, foi relatado que cerca de 5 milhões de pessoas entre os 20 e 59 anos vieram a óbito devido à diabetes. A fim de que se possa diminuir ou controlar os casos foi criado o Plano de Ações Estratégicas para o Enfrentamento das Doenças Crônicas não Transmissíveis (DCNT), cujo objetivo é o combate de doenças, como diabetes (Flor \& Campos, 2017).

Portanto, o presente trabalho justifica-se na necessidade de apresentar metodologias inovadoras no campo da saúde como, por exemplo, o uso de marcadores biológicos no acompanhamento de enfermidades. Além disso, auxilia no acréscimo e renovação de referência na literatura referente à diabetes e seu diagnóstico clínico.

\section{Metodologia}

Trata-se de uma pesquisa de caráter bibliográfica sistemática com abordagem qualitativa, de natureza básica, com objetivos exploratórios, pois baseia-se na apresentação e avaliação de uma alternativa no diagnóstico e monitoramento terapêutico da diabetes mellitus. Segundo Ercole, Melo e Alcoforado (2014), a revisão sistemática busca atender a um objetivo específico, realizando um levantamento detalhado da literatura e avaliando a eficácia da solução para a questão proposta na pesquisa. Trata-se de um estudo secundário por realizar um levantamento de resultados publicados em fontes primárias como, por exemplo, estudos observacionais ou ensaios clínicos randomizados (Galvão \& Pereira, 2014).

Koller e Hohendorff (2014) descreveram passos para a elaboração de uma revisão sistemática, dessa maneira serviram como suporte para realização da metodologia a seguir. Inicialmente, realiza-se a delimitação do tema e, em seguida, escolha das palavras-chave e dos bancos de dados, pesquisa e seleção de artigos, avaliação e por fim, síntese dos resultados obtidos. 
Os dados foram coletados através da busca de publicações na área. Como plataformas de busca foram empregadas a biblioteca Scientific Electronic Library Online (SciELO), a interface PUBMED e a base Literatura Latino-Americana e do Caribe em Ciências da Saúde (LILACS). O recorte temporal inclui os anos de 2010 à 2021.

Através do Descritores de Assunto em Ciências da saúde da BIREME (DECs) e do Medical Subject Headings (MESH), foram selecionadas as seguintes palavras chaves "1,5-anidroglucitol", "diabetes mellitus", "hemoglobina A glicada" e "peptídeo C". Para busca avançada foi definido que ao menos um dos termos estivesse presente no título, resumo ou como palavra-chave do artigo.

As palavras-chave foram combinadas nas línguas inglesa e portuguesa. A busca nas bases de dados ocorreu no período de setembro de 2020 à março de 2021. Para a busca avançada fez-se uso do operador booleano "AND”. Por exemplo, a busca na SciELO utilizou "diabetes mellitus (título)", "petídeo C AND diabetes mellitus (título)", "diabetes mellitus AND hemoglobina A glicada", "glycated hemoglobin". Na LILACS, foram pesquisados artigos somente com a palavra-chave "hemoglobina A glicada". E por fim, na PUBMED, "1,5-Anhydroglucitol (título/resumo)", "peptide c (termos mesh) AND diabetes".

Para a elaboração da pergunta da pesquisa e a busca nas bases de dados foi aplicada a estratégia do anagrama PICOS. Sendo assim, foi possível apurar melhor os dados encontrados. A seguir, a tabela contendo os componentes da pergunta da pesquisa (Tabela 1).

Tabela 1. Estratégia PICOS.

\begin{tabular}{ccc}
\hline DESCRIÇÃO & ABREVIAÇÃO & COMPONENTES DA \\
& PERGUNTA \\
\hline População & $\mathrm{P}$ & Pacientes diabéticos \\
Intervenção & $\mathrm{I}$ & Dosagem de biomarcadores \\
Comparação & $\mathrm{C}$ & Testes glicêmicos usuais \\
Desfecho & $\mathrm{O}$ & Resultados da eficácia da dosagem \\
Tipos de estudos & & dos biomarcadores \\
& $\mathrm{S}$ & Observacionais, experimentais e \\
\end{tabular}

Fonte: Galvão e Pereira (2014).

Como critérios de inclusão foram apontados artigos que tratem de pesquisa com grupos ou amostras de seres humanos com utilização de exames glicêmicos, podendo estes abordarem a dosagem de biomarcadores. Sendo assim, foram selecionados artigos observacionais, ensaios clínicos e estudos experimentais, publicados em inglês ou português. Os pacientes do estudo devem possuir idade igual ou superior aos 18 anos. Os artigos devem relatar a efetividade dos métodos utilizados em sua pesquisa.

Como critérios de exclusão, foram excluídas publicações em anais de eventos, artigos de revisão ou sem metodologia clara, artigos duplicados, conteúdos de livros, artigos que não estão na língua inglesa ou portuguesa ou que estejam fora do recorte temporal estabelecido na pesquisa. Além de documentos de difícil acesso tanto na forma online como impressa ou que estejam incompletos e artigos que não utilizem ao menos uma dosagem de biomarcadores da diabetes.

Os critérios de seleção e os resultados dos artigos selecionados para a discussão foram organizados através do fluxograma PRISMA statement 2020. A seleção dos estudos ocorreu por 3 fases, sendo estas a identificação dos estudos, a triagem ou seleção e a inclusão dos estudos na revisão. 
A seguir, foi determinado o nível de evidência dos estudos incluídos na revisão através da tabela de classificação do sistema GRADE. Logo depois, foi construído um quadro-resumo de própria autoria contendo os dados dos artigos selecionados para análise final, como autor e ano, objetivos e metodologia e, em seguida, foram discutidas as contribuições dos autores.

\section{Resultados e Discussão}

Inicialmente, foi realizada uma pesquisa nas plataformas de dados por meio das palavras-chave.

Figura 1. Fluxograma com seleção dos estudos.

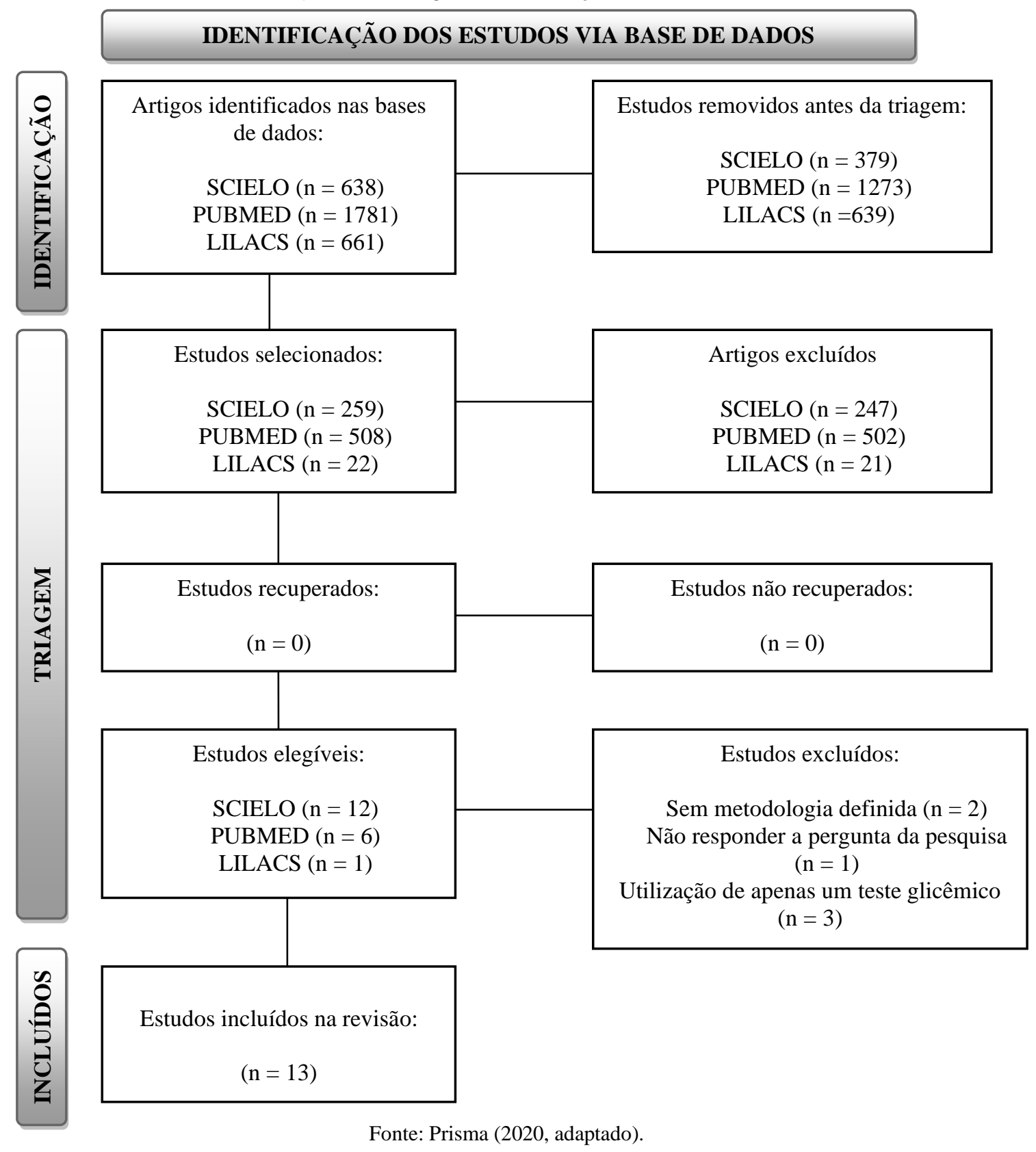

Em seguida, foram retirados artigos duplicados e inelegíveis, por não estarem no recorte temporal. Logo, foi realizada uma apuração por meio do título e critérios de exclusão e inclusão para que se adequasse melhor à pesquisa e, por fim, foram 
selecionados 19 estudos. Não houve recuperação de artigos. Destes, 6 foram retirados por não corresponderem a todos os critérios de inclusão. Por fim, 13 artigos foram selecionados e incluídos na pesquisa (Figura 1).

Após a seleção dos artigos, foram expostos os artigos que mais se adequassem ao propósito da pesquisa. Logo, é necessário avaliar a qualidade da evidência das fontes pesquisadas através da escala Níveis de Evidência Científica segundo a classificação do sistema GRADE (Quadro 1).

Quadro 1. Imagem com os Níveis de evidências de acordo com o sistema GRADE.

\begin{tabular}{|c|c|c|c|}
\hline Nível & Definição & Implicações & Fonte de informação \\
\hline Alto & $\begin{array}{l}\text { Há forte confiança } \\
\text { de que o } \\
\text { verdadeiro efeito } \\
\text { esteja próximo } \\
\text { daquele estimado. }\end{array}$ & $\begin{array}{l}\text { É improvável que } \\
\text { trabalhos adicionais } \\
\text { irãomodificar a } \\
\text { confiança na } \\
\text { estimativa do efeito. }\end{array}$ & $\begin{array}{c}\text { - Ensaios clínicos bem } \\
\text { delineados, com } \\
\text { amostrarepresentativa. } \\
\text { - Em alguns casos, } \\
\text { estudos observacionais } \\
\text { bem delineados, com } \\
\text { achados consistentes*. }\end{array}$ \\
\hline Moderado & $\begin{array}{l}\text { Há confiança } \\
\text { moderada no } \\
\text { efeito estimado. }\end{array}$ & $\begin{array}{c}\text { Trabalhos futuros } \\
\text { poderãomodificar a } \\
\text { confiança } \\
\text { na estimativa de } \\
\text { efeito,podendo, } \\
\text { inclusive, } \\
\text { modificar a } \\
\text { estimativa. } \\
\end{array}$ & $\begin{array}{l}\text { - Ensaios clínicos com } \\
\text { limitações leves**. } \\
\text { - Estudos observacionais } \\
\text { bemdelineados, com } \\
\text { achados consistentes*. }\end{array}$ \\
\hline Baixo & $\begin{array}{l}\text { A confiança no } \\
\text { efeito é } \\
\text { limitada. }\end{array}$ & $\begin{array}{l}\text { Trabalhos futuros } \\
\text { provavelmente } \\
\text { terão um impacto } \\
\text { importante em nossa } \\
\text { confiança na } \\
\text { estimativa de efeito. }\end{array}$ & $\begin{array}{l}\text { - Ensaios clínicos com } \\
\text { limitações moderadas**. } \\
\text { - Estudos observacionais } \\
\text { comparativos: coorte e } \\
\text { caso-controle. }\end{array}$ \\
\hline $\begin{array}{l}\text { Muito } \\
\text { Baixo }\end{array}$ & $\begin{array}{l}\text { A confiança na } \\
\text { estimativa de } \\
\text { efeito é muito } \\
\text { limitada. Há } \\
\text { importante grau } \\
\text { de incerteza nos } \\
\text { achados. } \\
\end{array}$ & $\begin{array}{l}\text { Qualquer estimativa de } \\
\text { efeito é incerta. }\end{array}$ & $\begin{array}{c}\text { - Ensaios clínicos com } \\
\text { limitações graves**. } \\
\text { - Estudos } \\
\text { observacionais } \\
\text { comparativos presença } \\
\text { delimitações**. } \\
\text { - Estudos observacionais não } \\
\text { comparados***. } \\
\text { - Opinião de especialistas. }\end{array}$ \\
\hline
\end{tabular}

* Estudos de coorte sem limitações metodológicas, com achados apresentando tamanho de efeito grande e/ou gradiente dose-resposta.

**Limitações: vieses no delineamento do estudo, inconsistência nos resultados, desfechos substitutos ou validade externa comprometida.

*** Séries e relatos de casos.

Fonte: Elaboração Grade (2014, adaptado).

Através da imagem acima (Quadro 1), é possível analisar a qualificação de estudos conforme o nível de evidência apresentado por estes. Os artigos incluídos na revisão apresentam nível de alto a baixo de evidência por tratar-se de estudos observacionais e ensaios com limitações ou bom delineamento e achados consistentes.

No Quadro 2, podem-se observar os artigos incluídos na revisão, seus objetivos e metodologia para a pesquisa. Além disso, contêm seus dados básicos como autor e ano. Os dados encontram-se organizados por ordem alfabética pelo nome do autor. 
Quadro 2. Dados dos artigos: Autor/ano, objetivos e metodologia dos estudos.

\begin{tabular}{|c|c|c|}
\hline AUTOR/ANO & OBJETIVOS & METODOLOGIA \\
\hline $\begin{array}{l}\text { (Cavagnolli, Gross } \\
\text { \& Carmargo, 2010) }\end{array}$ & $\begin{array}{l}\text { Analisar a concordância diagnóstica entre GJ, TOTG } \\
\text { e A1C no diagnóstico de DM. }\end{array}$ & $\begin{array}{l}\text { Ensaio com } 498 \text { indivíduos acima de } 18 \text { anos que } \\
\text { foram submetidos a realização do TOTG. }\end{array}$ \\
\hline (Franco et al., 2019) & $\begin{array}{l}\text { Analisar resultados de glicemia de jejum de pacientes } \\
\text { da rede pública e sua correlação com hemoglobina } \\
\text { glicada e lipídios }\end{array}$ & $\begin{array}{l}\text { Estudo transversal, com } 77.581 \text { pacientes, atendidos } \\
\text { em 2014, com } 18 \text { anos ou mais. Realização da GJ, } \\
\text { HbA1c, colesterol total e frações, triglicérides e } \\
\text { albuminúria. }\end{array}$ \\
\hline $\begin{array}{c}\text { (Fujimoto et al., } \\
\text { 2016) }\end{array}$ & $\begin{array}{l}\text { Avaliar se há correlação das dosagens de frutosamina } \\
\text { e de hemoglobina glicosilada (HbA1c) com as } \\
\text { frequências de desvios de glicemia capilar em } \\
\text { gestantes com diabetes mellitus. }\end{array}$ & $\begin{array}{l}\text { Estudo observacional, retrospectivo, de corte } \\
\text { transversal, envolvendo gestantes portadoras de } \\
\text { diabetes gestacional ou pré-gestacional que iniciaram } \\
\text { o pré-natal. Realizou-se monitoramento da glicemia, } \\
\text { frutosamina e HbAlc. }\end{array}$ \\
\hline (Iser et al., 2021) & $\begin{array}{l}\text { Avaliar a prevalência de hiperglicemia intermediária } \\
\text { e pré-diabetes em adultos brasileiros, considerando } \\
\text { diferentes critérios diagnósticos, e estabelecer fatores } \\
\text { associados à sua ocorrência. }\end{array}$ & $\begin{array}{l}\text { Análise de dados do PNS entre } 2014 \text { e } 2015 \text { com } \\
60.202 \text { moradores para dosagem de hemoglobina } \\
\text { glicada e colesterol. }\end{array}$ \\
\hline (Ma et al., 2015) & $\begin{array}{l}\text { Investigar as associações de dois marcadores } \\
\text { glicêmicos não tradicionais, albumina glicada (GA) e } \\
\text { 1,5-anidroglucitol (1,5-AG), bem como hemoglobina } \\
\text { glicada A1c (HbA1c) com doença arterial } \\
\text { coronariana. }\end{array}$ & $\begin{array}{l}\text { Foi realizado um ensaio com } 278 \text { indivíduos } \\
\text { submetidos a cinecoronagrafia e rastreio de } 1,5 \mathrm{Ag} \text { e } \\
\text { HbA1c. }\end{array}$ \\
\hline $\begin{array}{c}\text { (Menezes et al., } \\
\text { 2012) }\end{array}$ & $\begin{array}{l}\text { Verificar possíveis variações na concentração de } \\
\text { hemoglobina }(\mathrm{Hb}) \text { glicada (HbA1c) na presença de } \\
\text { Hbs S e C e avaliar o impacto da redução da HbAlc } \\
\text { na avaliação clínica e no monitoramento do paciente } \\
\text { diabético. }\end{array}$ & $\begin{array}{l}\text { Ensaio com } 150 \text { indivíduos diabéticos, de ambos os } \\
\text { gêneros, com idade média de } 56 \text { anos. Foram } \\
\text { determinadas a glicemia de jejum e a HbA1c por } \\
\text { metodologia de oxidase-peroxidase e cromatografia } \\
\text { líquida de alta eficiência (CLAE). }\end{array}$ \\
\hline (Selvin et al., 2016) & $\begin{array}{l}\text { Avaliar associações de } 1,5-\mathrm{AG} \text { com desfechos } \\
\text { cardiovasculares em longo prazo em um ambiente de } \\
\text { base populacional. }\end{array}$ & $\begin{array}{l}\text { Ensaio com dosagem de } 1,5-\mathrm{AG} \text { em } 11.106 \\
\text { participantes para avaliar Risco de Aterosclerose em } \\
\text { Comunidades (ARIC) e examinar associações } \\
\text { prospectivas com doença cardíaca coronária, acidente } \\
\text { vascular cerebral isquêmico, insuficiência cardíaca e } \\
\text { morte ao longo de } 20 \text { anos. }\end{array}$ \\
\hline (Selvi & $\begin{array}{l}\text { Definir os intervalos de referência e as diferenças } \\
\text { demográficas na frutosamina, albumina glicada e } 1,5- \\
\text { AG por meio do uso de dados de uma população } \\
\text { americana bem caracterizada de adultos negros e } \\
\text { brancos. }\end{array}$ & $\begin{array}{l}\text { Ensaio com dosagem de frutosamina, albumina } \\
\text { glicada e 1,5-AG em uma amostra comunitária de } \\
\text { adultos negros e brancos norte-americanos que } \\
\text { participaram do Estudo de Risco de Aterosclerose em } \\
\text { Comunidades. }\end{array}$ \\
\hline (Serfaty & $\begin{array}{l}\text { Avaliar se anti-GAD positivo e PC detectável se } \\
\text { correlacionam com a presença de outras doenças } \\
\text { autoimunes, com controle glicêmico e com risco de } \\
\text { retinopatia no diabetes melito tipo } 1 \text { (DMT1) > } 3 \\
\text { anos de duração. }\end{array}$ & $\begin{array}{l}\text { Ensaio clínico com } 50 \text { pessoas com DMT1 } \\
\text { entrevistadas realizaram fundoscopia e dosaram PC } \\
\text { pré e pós-glucagon, HbA1C e anti-GAD. }\end{array}$ \\
\hline 2016) & $\begin{array}{l}\text { Testar se uma elevação na proporção sérica de pró- } \\
\text { insulina para peptídeo C (PI: C), um biomarcador de } \\
\text { disfunção do retículo endoplasmático (ER) de células } \\
\beta \text {, estava associada à progressão para diabetes tipo } 1 \text {. }\end{array}$ & $\begin{array}{l}\text { Ensaio com medição dos níveis totais de PI e C em } \\
\text { jejum em amostras de soro obtidas de participantes, } \\
\text { um coorte de parentes autoanticorpos positivos sem } \\
\text { diabetes de indivíduos com diabetes tipo } 1 \text {. }\end{array}$ \\
\hline $\begin{array}{c}\text { (Tavares et al., } \\
\text { 2017) }\end{array}$ & $\begin{array}{l}\text { Avaliar os níveis de hemoglobina glicada (HbAlc) } \\
\text { em pacientes heterozigotos para variantes de } \\
\text { hemoglobina e comparar os resultados desse teste } \\
\text { com os de um grupo controle. }\end{array}$ & $\begin{array}{l}\text { Estudo experimental baseado na comparação de } \\
\text { testes de HbA1c em duas populações diferentes, com } \\
\text { um grupo teste representado por indivíduos } \\
\text { heterozigotos para variantes da hemoglobina (AS e } \\
\text { AC) e um grupo controle formado por pessoas com } \\
\text { perfil eletroforético AA. }\end{array}$ \\
\hline $\begin{array}{c}\text { (Uzunlulu et al., } \\
\text { 2019) }\end{array}$ & $\begin{array}{l}\text { Determinar as reservas de células } \beta \text { de pacientes com } \\
\text { diabetes tipo } 2 \text { que são tratados com insulina usando } \\
\text { concentrações de peptídeo } C \text { em jejum e investigar as } \\
\text { características clínicas relacionadas às concentrações } \\
\text { de peptídeo C. }\end{array}$ & $\begin{array}{l}\text { Ensaio clínico com pacientes com diabetes tipo 2, que } \\
\text { usavam insulina como monoterapia ou em terapia } \\
\text { combinada, foram divididos em três grupos }\end{array}$ \\
\hline $\begin{array}{c}\text { (Varaschim et al., } \\
2020)\end{array}$ & $\begin{array}{l}\text { Verificar as alterações do peso e índice de massa } \\
\text { corporal em pacientes obesos grau II e III com } \\
\text { diabete melito tipo } 2 \text { nos períodos pré e pós- } \\
\text { operatório e as alterações dos parâmetros } \\
\text { laboratoriais de glicemia de jejum, glicemia pós- } \\
\text { prandial, hemoglobina glicada, insulina nos períodos } \\
\text { pré e pós-operatório. }\end{array}$ & $\begin{array}{l}\text { Estudo prospectivo com } 40 \text { indivíduos com obesidade } \\
\text { grau I e II realizou-se uma análise do pré e pós- } \\
\text { operatórios com testes de glicemia em jejum, IMC, } \\
\text { HbA1c, insulina e glicemia pós-prandial. }\end{array}$ \\
\hline
\end{tabular}


A partir do Quadro 2, pôde-se analisar as principais propostas dos autores, a seguir, encontra-se a revisão dos artigos incluídos através dos resultados e conclusão destes.

Cavagnolli, Gross e Camargo (2010) realizaram um ensaio para diagnosticar diabetes mellitus através dos testes de glicemia em jejum, TOTG e HbAlc. Os indivíduos diagnosticados diretamente pela hemoglobina glicada já tinham idade avançada e problemas cardiovasculares resultantes da DM. Foi analisado que esse marcador apresenta baixa sensibilidade. Por outro lado, avaliou-se que o diagnóstico por TOTG detectou um grupo maior de indivíduos do que quando realizado por GJ. Resumindo, os três testes identificam números diferentes de diabéticos, ou seja, populações diferentes e para que o diagnóstico seja preciso é necessário correlacionar os testes.

Franco et al. (2019), apresentaram em seu estudo transversal pacientes que monitoravam a DM via glicemia em jejum e dosagem de hemoglobina glicada. Estabeleceu-se que a idade avançada em pacientes do sexo masculino estava interligada com uma maior alteração nos exames. Os autores demonstraram que em alguns casos, a $\mathrm{HbA1c}$ tem sido mais importante tanto no rastreio quanto diagnóstico. No entanto, em quadros de pré-diabetes não apresenta-se tão eficaz. Além disso, grande parte dos pacientes obtiveram altas taxas de colesterol total e suas frações.

$\mathrm{O}$ uso da hemoglobina glicada como principal meio de diagnóstico ainda gera discordância entre a Organização Mundial de Saúde (OMS) e a Associação Americana de Diabetes (ADA). Porém, foi observado no estudo que o controle glicêmico via GJ não estava sendo viável, além de que a pouca busca por frações da albumina reflete a baixa pesquisa por complicações advindas da DM, logo o uso da hemoglobina glicada torna-se necessário (Franco et al., 2019).

Tratando-se de quadros de diabetes gestacional, Fujimoto et al. (2016), avaliou o monitoramento glicêmico e obteve resultados significativos no uso da HbA1c para casos de hiperglicemia de longa duração. No entanto, a dosagem por esse biomarcador mostra-se limitada em quadros de picos hiperglicêmicos inferiores há 120 dias. Além disso, esse método sofre interferências da taxa de renovação das hemácias tanto em anemias quanto em hemólises.

Os autores utilizaram a frutosamina quando a hemoglobina glicosilada estivesse limitada, avaliando os casos com menor intervalo de tempo. Mesmo com o uso da frutosamina ainda é preferível o uso da glicemia capilar para controle da DM gestacional por automonitoramento. Por fim, ainda é necessário avaliar melhor como os biomarcadores podem ser eficazes no controle de diabetes em gestantes (Fujimoto et al., 2016).

Para Iser et al. (2021), a ocorrência de pré-diabetes e hiperglicemia intermediária ocorria por ao menos 3 fatores de risco, além de determinantes ligados a raça ou características socioeconômicas. Os autores relataram que cerca de 7,5\% da população tem alterações glicêmicas. Através da hemoglobina glicada foi possível estabelecer distinção racial, sendo possível com esse marcador detectar as diferenças populacionais. Além disso, foi determinado que pacientes com TOTG e GJ alterados eram na maioria mais velhos e com predisposição para desenvolver diabetes.

Destacou-se que o uso de HbA1c é limitado por ter sensibilidade questionável, cerca de 49\%, além de não ser adequado para monitoramento de picos glicêmicos em curto prazo. Logo, ainda são necessárias buscas de testes específicos para pré-diabetes e hiperglicemia intermediária, pois até mesmo o TOTG é questionável por não possuir um largo alcance diagnostico (Iser et al., 2021).

Outro fator limitante na dosagem de HbA1c, é a presença de hemoglobinas variantes. Menezes et al. (2012), encontraram alterações da $\mathrm{HbAlc}$ em portadores de hemoglobina variante AC e AS, em quadros de hemoglobinopatias, ocasionando na redução do biomarcador. Dessa forma, os resultados da glicemia podem ser interpretados erroneamente. Sendo assim, os exames devem ser avaliados corretamente para que se possa evitar diagnósticos incorretos.

Os pacientes com hemoglobinas variantes e diabéticos vão apresentar alterações nos exames glicêmicos, mas a HbAlc permaneceu normal. Por esse motivo, foi considerado que os resultados estavam superestimados. Logo, tem sido necessário a substituição da metodologia usual por essa não conseguir identificar os interferentes das hemácias e apresentar 
valores corretos. Além disso, a redução do volume de hemoglobina $(\mathrm{Hb})$ é outro interferente. Desse modo, devem ser considerados os valores de Hbs em mulheres e homens e suas alterações quanti-qualitativas (Tavares et al., 2017).

Recentemente, os biomarcadores foram associados à busca de complicações advindas da diabetes mellitus como a Doença Arterial Crônica (DAC). No estudo de Ma et al. (2015), 194 participantes diagnosticados com DAC apresentaram nos exames detectadas altas taxas de HbA1c, AG e baixos níveis de 1,5 AG. Os fatores de risco relatados, além da hiperglicemia contínua, foram glicemia pós-prandial elevada e o elevado estresse oxidativo. Concluiu-se que a albumina glicada é o marcador com maior poder de detecção de DAC por ser um preditor da aterosclerose e acelerar complicações microvasculares.

Selvin et al. (2016) aprofundaram-se na relação entre o 1,5 anidroglucitol e as complicações cardiovasculares e a mortalidade por diabetes em indivíduos com idade avançada. Algumas das condições associadas foram acidente vascular cerebral isquêmico, insuficiência cardíaca e o óbito. Dos pacientes do estudo, aqueles com 1,5 AG em concentrações inferiores a $6 \mu \mathrm{g} / \mathrm{mL}$ estavam mais propensos a adquirirem doenças coronarianas. Ademais, é possível concluir que o 1,5 AG pode ser usado no monitoramento de complicações em longo prazo, mas ainda é estudada a sua aplicação no controle da diabetes.

Em casos de cirurgias para resolução de comorbidades associadas a DM como a obesidade, é necessário monitoramento contínuo da glicemia. No estudo de Varaschim et al. (2012) foi relatado que a HbA1c e a glicemia em jejum não são adequados para avaliação glicêmica do pré e pós-operatório de pacientes obesos por derivação gastrojejunal em Y-deRoux. Como substituto, o uso do exame de glicemia pós-prandial pode ser mais adequado. Dessa maneira, é possível concluir que os exames glicêmicos não só apresentam poder de confirmação diagnóstica, mas são parâmetros avaliados em cirurgias.

Por serem marcadores descobertos a pouco tempo, a albumina glicada e o 1,5 anidroglucitol ainda não possuem valores de referência bem delimitados. Por isso, Selvin et al. (2018), determinaram por meio de uma média com ensaios clínicos que para a albumina glicada os valores normais estabelecidos situavam-se entre 11,9 a 15,8\%, em pessoas negras a concentração no corpo é mais elevada em relação aos brancos. Alguns indivíduos com valores próximos de 13,6\% poderiam ter quadros de pré-diabetes.

Tratando-se do 1,5 AG, sua concentração reflete quando a glicemia ultrapassa o limiar renal. Poucos pacientes apresentaram alterações nesse marcador, mas os autores estabeleceram que valores menores que $10 \mu \mathrm{g} / \mathrm{mL}$ (microgramas por mililitro) são indicativos de casos hiperglicêmicos. Portanto, pode-se verificar que esses dois marcadores biológicos ainda apresentam mecanismos desconhecidos, mas que podem ser aplicados como substitutos do HbA1c no diagnóstico e monitoramento de diabetes revolucionando a prática clínica.

Para pesquisa específica de diabetes mellitus tipo 1, utilizam-se normalmente anticorpos como o anti-GAD. Além dos marcadores já citados, existe também um mais tradicional, o peptídeo C (PC). Este é um derivado do metabolismo da insulina e é liberado em concentrações equivalentes as do hormônio. Esses dois marcadores positivando por um longo tempo induzem à formação de doenças autoimunes (Serfaty et al., 2010).

Serfarty et al. (2010) também explicaram que o anti-GAD, quando positiva tardiamente pode ser indicativo de um sistema imune deprimido. Logo esse anticorpo e o peptídeo $\mathrm{C}$ podem ser utilizados não só no diagnóstico como também na avaliação do desenvolvimento de comorbidades autoimunes como, por exemplo, problemas tireoidianos.

Além disso, o peptídeo C também pode ser utilizado para predizer o risco da diabetes recém-descoberta. Sims et al. (2016) demonstraram que o aumento simultâneo do PC com pró-insulina pode definir o risco de diabetes detectada em menos de 1 ano. Essa elevação é observada não só na diabetes tipo 1 como no tipo 2. Ainda mais, a razão PC e pró-insulina pode identificar a disfunção de células beta-pancreáticas. É importante ressaltar que esse aumento em crianças pode desencadear reação autoimune grave.

Em uma análise realizada por Uzunlulu et al. (2019), foram estabelecidos os limites adequados de peptídeo C, os valores devem situa-se entre 0,5 a $3,2 \mathrm{ng} / \mathrm{mL}$. Ademais, foi verificado que diabéticos tipo 2 deveriam utilizar o tratamento via 
insulina e que o peptídeo C seria o determinante para a avaliação do tratamento. Além disso, o marcador auxiliaria na redução de quadros de hipoglicemia e aumento de peso derivados do tratamento.

\section{Conclusão}

Com base nos dados apresentados, pôde-se averiguar que, mesmo sendo recente, o uso dos biomarcadores vem ganhando destaque nas análises clínicas. São específicos nas dosagens a curto, médio e longo prazo, não necessitando que o paciente realize jejum, além de apresentar boa confiabilidade.

Devido às limitações diagnósticas dos exames glicêmicos usuais, os biomarcadores têm sido uma metodologia compensatória. São importantes não só na detecção do tipo de diabetes mellitus, como também na avaliação do risco de desenvolvimento de doenças microvasculares, acompanhamento de cirurgias e na eficácia da insulinoterapia.

Os biomarcadores possibilitam a detecção do tipo de diabetes que o indivíduo porta, por exemplo, a presença de autoanticorpos como o anti-GAD são indicativos da diabetes tipo 1. No entanto, os outros marcadores estão presentes no tipo 1 e 2, mesmo que na maioria dos casos sejam de diabetes tipo 2.

No entanto, eles não são exigidos como testes únicos, ou seja, sempre vão vir acompanhados de algum teste glicêmico como a glicemia em jejum, glicemia pós-prandial ou teste oral de tolerância. Dessa maneira, os autores ressaltam que os biomarcadores como a hemoglobina glicada, a albumina glicada, o 1,5-anidroglucitol e o peptídeo C são bons no monitoramento. Porém, no diagnóstico precoce priorizam-se os testes glicêmicos usuais.

Outro fator que limita o uso principal desses marcadores biológicos é o alto custo da análise, por serem necessários equipamentos mais sofisticados como no caso da dosagem de hemoglobina glicosilada. Em quadros de diabetes gestacional ou pré-diabetes ainda são necessários mais estudos para entender como os biomarcadores funcionam e como correlacionar estes com o diagnóstico. Por exemplo, a HbA1c ainda não é precisa em gestantes na marcação das flutuações ou não mensura picos hiperglicêmicos de menos de 3 meses.

Por fim, respondendo ao objetivo da pesquisa, os biomarcadores são uma metodologia alternativa no monitoramento. Porém, ainda é necessário aumentar a aplicação na prática clínica dos novos marcadores como o 1,5 anidroglucitol. E, além disso, eles são eficazes no controle e monitoramento terapêutico, bem como no rastreio tardio.

Dessa maneira, trabalhos futuros podem avaliar e validar métodos para avaliação dos biomarcadores e verificar se a aplicação destes pode substituir os métodos padrões para avaliação da glicemia. Sendo assim, pode ser possível comprovar experimentalmente os fatos abordados nesta revisão. Além disso, são importantes pesquisas acerca de possíveis novos biomarcadores e sua utilização em quadros de diabetes mellitus gestacional.

\section{Agradecimentos}

Agradeço a Deus por me iluminar, me dar força e nunca me deixar esquecer o caminho. Sem ele eu nunca estaria aqui. Aos meus pais, por nunca me deixarem desistir e me apoiarem nos estudos, me fornecendo o melhor que eu poderia ter. Aos meus amigos, Éryca e Júnior, por acreditarem em meu potencial e me instruírem nessa jornada. Agradeço, pelo apoio e por sempre me ouvirem quando eu precisei. Obrigada por não saírem do meu lado. Ao meu orientador, Prof. Jardes, por ter tido paciência comigo e me guiar nesses meses de construção do projeto, além de mostrar que sou capaz de tudo. E por fim, a todos que de alguma forma participaram dessa conquista, me incentivando e me dando forças para seguir em frente. 


\section{Referências}

American Diabetes Association (2014). Diagnosis and classification of Diabetes Mellitus. Diabetes Care, 37(1), 81-90. https://doi.org/10.2337/dc14-S081.

Cavagnolli, G., Gross J. L. \& Camargo, J. L. (2010). HbA1C, Glicemia de jejum e teste oral de tolerância à glicose no diagnóstico de diabetes: que teste usar? Rev. HCPA, 30(4), 315-320. https://pesquisa.bvsalud.org/portal/resource/pt/biblio-834384.

Durnwald, C. P., Mele, L., Spong, C. Y., Ramin, S. M., Varner, M. W., Rouse, D. J., Sciscione, A., Catalano, P., Saade, G., Sorokin, Y., Tolosa, J. E., Casey, B. \& Anderson, G. D. (2011). Glycemic characteristics and neonatal outcomes of women treated for mild gestacional diabetes. Obstet. Gynecol. 117(4), 819827. https://doi.org/10.1097 AOG.0b013e31820fc6cf.

Ercole, F. F., Melo, L. S. \& Alcoforado, C. L. G. C. (2014). Revisão integrativa versus revisão sistemática. Rev. Min. Enferm. 18(1), 9-11. http://www.dx.doi.org/10.5935/1415-2762.20140001.

Flor, L. S. \& Campos, M. R. (2017). Prevalência de diabetes mellitus e fatores associados na população adulta brasileira: evidências de um inquérito de base populacional. Rev. Bras. Epidemiol., 20(1), 16-29. https://doi.org/10.1590/1980-5497201700010002.

Franco, L. F., Mafra, A. C. C. N., Bracco, M. M., Franco, L. J., Naves, L. K., Ribeiro, G. M. F. \& Mangueira, C. L. P. (2019). Glicemia de jejum em pacientes da rede pública de saúde na região Sul de São Paulo: correlação com hemoglobina glicada e níveis lipídicos. Rev. Bras. Epidemiol, 22 (190058), 1-13. https://doi.org/10.1590/1980-549720190058.

Fujimoto, C. Y., Costa, R. A., Zaccara, T. A., Paganotti, C. F. \& Francisco, R. P. V. (2016). Correlação das dosagens de frutosamina e de hemoglobina glicosilada com o perfil glicêmico em gestantes com diabetes mellitus. Rev. Bras. Ginec., 38(1), 20-26. https://doi.org/10.1055/s-0035-1570108.

Galvão, T. F. \& Pereira, M. G. (2014). Revisões sistemáticas da literatura: passos para a sua elaboração. Epidemiol. Serv. Saúde. 23(1), 183-184. http://dx.doi.org/10.5123/S1679-49742014000100018.

Iser, B. P. M., Pinheiro, P. C., Malta, D. C., Duncan, B. B. \& Schimidt, M. I. (2021). Prevalência de pré-diabetes e hiperglicemia intermediária em adultos e fatores associados: Pesquisa Nacional de Saúde. Ciênci. Saúde Colet., 26(2), 531-540. https://doi.org/10.1590/1413-81232021262.34852020.

Koller, S. H., Couto, M. C. P. P. \& Hohendorff, J. V. (2014). Manual de produção científica (Cap. 3, pp. 55-70). Penso.

Ma, X., Hu, X., Zhou, J., Hao, Y., Luo, Y., Lu, Z., Bao, Y. \& Jia, W. (2015). Glycated albumin is more closely correlated with coronary artery than 1,5anhydorglucitol and glycated hemoglobin A1c. Cardiovasc. Diabetol., 14(16), 1-7. https://doi.org/10.1186/s12933-014-0166-z.

Marques, R. M. B., Fornes, N. S. \& Sstringhini, M. L. F.(2011). Farores socioeconômicos, demográficos, nutricionais e de atividade física no controle glicêmico de adolescentes portadores de diabetes melito tipo 1. Arq. Bras. Endocrinol. Metab. 5(3), 194-202. https://doi.org/10.1590/S000427302011000300004

Menezes, M. G. S., Couto, F. D., Santos Junior, L. S., Adórno, E. V., Barbosa, C. G., Gonçalves, M. S. \& Couto, R. D. (2012). Determinação de HbA1c por CLAE: interferência de variantes de hemoglobinas S e C e alta concentração de HbF. J. Bras. Patol. Med. Lab., 48(5), 337-344. https://doi.org/10.1590/S 1676-24442012000500006.

Ministério da Saúde (2014). Diretrizes Metodológicas Sistema GRADE - manual de graduação da qualidade da evidência e força de recomendação para tomada de decisão em saúde. Editora MS, 2014, 74p.

Prisma (2020). Prisma-statement.org. http://prisma-statement.org/prismastatement/flowdiagram.aspx.

Rossaneis, M. A., Andrade, S. M., Gvozd, R., Pissinati, P. S. C. \& Haddad, M. C. L. (2019). Fatores associados ao controle glicêmico de pessoas com diabetes mellitus. Ciência \& Saúde Coletiva, 24(3), 997-1005. https://doi.org/10.1590/1413-81232018243.02022017.

Sá, R. C, Navas, E. A. F. A. \& Aalves, S. R. (2014). Diabetes mellitus: Avaliação e controle através da glicemia em jejum e hemoglobina glicada. Revista Univap, 20(35), 15-23. http://dx.doi.org/10.18066/revunivap.v20i35.129.

Sociedade Brasileira de Diabetes-SBD (2019-2020). Diretrizes da Sociedade Brasileira de Diabetes. SBD.

Selvin, E., Rawlings, A., Lutsey, P., Maruthur, N., Pankow, J. S., Steffes, M. \& Coresh, J. (2016). Association of 1,5-Anhydroglucitol With Cardiovascular Disease and Mortality. Diabetes, 65(1), 201-208. https://doi.org/10.2337/db15-0607.

Selvin, E., Warren, B., He, X., Sacks, D. B. \& Saenger, A. K. (2018). Establishment of Community-Based Reference Intervals for Fructosamine, Glycated Albumin and 1,5-Anhydorglucitol. Clinical Chemistry, 64(5), 843-850. https://doi.org/10.1373/clinchem.2017.285742.

Serfaty, F. M., Dantas, J. R., Almeida, M. H., Duarte, J. D. G., Kupfer, R., Campos, F., Zajdenverg, L., Milech, A., Rodacki, M. \& Oliveira, J. E. P. (2010). Implicações clínicas da persistência de anti-GAD positivo e peptídeo C detectável em pacientes com diabetes melito tipo 1 de longa duração. Arq. Bras. Endocrinol. Metab., 54(5), 449-454. https://doi.org/10.1590/S0004-27302010000500004.

Silva, G. A., Souza, C. L. \& Oliveira, M. V. Teste oral de tolerância à glicose: solicitações desnecessárias e condições adequadas a realização do teste. J. Bras. Patol. Med. Lab., 56(0932020), 1-7. https://doi.org/10.5935/1676-2444.20200010.

Sims, E. K., Chaudhry, Z., Watkns, R., Syed, F., Blum, J., Ouyang, F., Perkins, S. M., Mirmira, R. G., Sosenko, J., DiMeglio, L. A. \& Evans-Molina, C. (2016). Elevations in the fasting serum proinuslin-to-C-peptide ratio precede the onset of type 1 diabetes. Diabetes Care, 39(9), $1519-1526$. https://doi.org/10.2337/dc15-2849.

Tavares, R. S., Souza, F. O., Francescantonio, I. C. C. M., Soares, W. C. \& Mesquita, M. M. (2017). HBA1C levels individuals heterozygous for hemogloin variants. Rev. Assoc. Med. Bras., 63(4), 341-346. https://doi.org/10.1590/1806-9282.63.04.341. 
Research, Society and Development, v. 10, n. 10, e135101018736, 2021

(CC BY 4.0) | ISSN 2525-3409 | DOI: http://dx.doi.org/10.33448/rsd-v10i10.18736

Uzunlulu, M., Oguz, A., Bahadir, M. A., Erbakan, A. N., Keskinler, M. V. \& Mesci, B. A. (2019). C-peptide concentrations in patients with type 2 treated with insulin. Diabetes Metab. Syndr, 13(6), 3099-3104. https://doi.org/10.1016/j.dsx.2019.11.010.

Varaschim, M., Nassif, P. A. N., Moreira, L. B., Nascimento, M. M., Vieira, G. M. N., Garcia, R. F., Sue, K. M. \& Cruz, M. A. (2012). Alterações dos parâmetros clínicos e laboratoriais em pacientes obesos com diabetes melito tipo 2 submetidos à derivação gastrojejunal em y de Roux sem anel. Rev. Col. Bras. Cir., 39(3), 178-182. https://doi.org/10.1590/S0100-69912012000300003. 